NOTA

$251-268$

\title{
EL PROBLEMA DE LA CONSTITUCIÓN DE LA INTERSUBJETIVIDAD EN EL PENSAMIENTO DE HUSSERL
}

The problem of the constitution of intersubjectivity in Husserl's thinking

Cristóbal Balbontín*

La teoría de la intersubjetividad, desarrollada por Husserl en sus Meditaciones Cartesianas, da la oportunidad para desmontar el mecanismo que equilibra en una falsa armonía el idealismo y el realismo implícito en su filosofía. La Quinta meditación cartesiana, que aborda el problema del otro en un momento de acentuado idealismo, revela como una contradicción la tracción a la que está sujeta la experiencia del otro entre dos tendencias: restituir el otro en mí y constituirlo como otro. Paradoja que está latente en toda su filosofía y que lleva a que la Quinta meditación cartesiana "estalle" sin proporcionar una respuesta satisfactoria ${ }^{1}$.

Sabemos que al publicarse sus Cartesianischen Meditationen Husserl era un pensador que había alcanzado cierta notoriedad y sus textos circulaban en el ambiente académico con particular interés. En dicha época nos encontramos con un Husserl ya maduro cuyo método de pensamiento, la fenomenología, se había aquilatado alcanzando un cierto reconocimiento. En virtud de ello, para dicha época se habían formulado las primeras críticas a su pensamiento, acusándolo de solipsismo trascendental. Él mismo hace referencia a tales críticas en sus Meditaciones: "Cuando yo el que medito, por medio de una epoye fundamental fenomenológica me reduzco a mi absoluto ego trascendental. ¿No me he convertido por ello en solus ipse y no lo sigo siendo mientras bajo el título de fenomenología efectúo una consecuente explicitación de mi mismo?" (Husserl, Meditaciones, 119). Con este propósito, en las Meditaciones cartesianas, Husserl no solo intenta realizar una exposición clara y concentrada de su pensamiento, sino que fundamentalmente se hace cargo de esta crítica realizada en defensa de sus ideas. Es precisamente en dicho espíritu que Husserl aborda el problema de lo social en Descubrimiento de la esfera del ser trascendental como intersubjetividad monádica.

\footnotetext{
${ }^{1}$ Desde un punto de vista formal la intersubjetividad es tratada expresamente por Husserl en la Quinta Meditación cartesiana y en el capítulo IV de las Ideas directrices para una fenomenología y una fenomenología pura, Libro II, bajo el título Constitución de la realidad anímica en la empatía. Del mismo modo el parágrafo 96 de Lógica formal y lógica trascendental bosqueja el tema bajo el título Problemáticas trascendentales de la intersubjetividad y el modo intersubjetivo, donde Husserl resume los puntos de referencia del tratamiento del problema de la intersubjetividad. No obstante, por su profundidad y desarrollo sistemático, es en la Quinta meditación Cartesiana donde el problema recibe su elaboración sistemática más profunda. Será entonces este texto el que nos servirá de objeto de estudio. Dejamos al margen así no solo los otros dos textos, sino también los manuscritos tardíos referidos al tema contenidos en los tomos XIV y XV de la Husserliana.
} 


\section{Cristóbal Balbontín}

I. EXPOSICIÓN DE LA TEORÍA TRASCENDENTAL DE LA CONSTITUCIÓN DE LA INTERSUBJETIVIDAD DE HUSSERL

Husserl comienza su exposición enfrentando directamente la objeción realizada a su pensamiento. Concretamente, el maestro de Gotinga se plantea que si la reducción trascendental solo liga a las corrientes puras de vivencias de la conciencia, como vivencias de un ego trascendental, ¿cómo es posible la experiencia de otros egos que van más allá de las puras cogitaciones de un ego solipsista? Para avanzar en dicha cuestión, Husserl decide recurrir como exigencia metodológica, a la epoye por medio de la que se hace abstracción de todas las efectuaciones constituidas de la intencionalidad referida, en este caso, de modo mediato o inmediato a una subjetividad extraña. Debido a que la intencionalidad de la conciencia, incluso cuando está dirigida al otro o extraño, ocurre en la exclusiva propiedad de un ego, es entonces en ella, en la intencionalidad en tanto mención de una conciencia lanzada como un rayo a la exterioridad, donde deben quedar develados los resortes sintéticos que hacen posible la constitución del otro en la subjetividad trascendental. Así, la constitución del otro pasa por un emplazamiento de la conciencia como faro dador de sentido y, en particular, del sentido del otro. Truismo donde se resalta el diálogo de lo "interior" y lo "exterior" a mí; diálogo en el cual se destaca el protagonismo del cuerpo en la función constitutiva del mundo.

En efecto, es por medio de mi cuerpo que yo me hallo inserto en el mundo que se me ofrece como espectáculo. Como señala Merleau-Ponty de forma repetida en la Phénoménologie de la Perception, de ello obtengo una perception mondanéisante. El cuerpo propio como nexo con la naturaleza y el mundo constituye entonces el punto cero de mis percepciones en el espacio y el tiempo y, como habíamos dicho, me es dado como parte fundamental de mi ser en su mención "soy" y que revela, en virtud de ese nexo, la participación del yo en cuanto hombre en el mundo. En otras palabras, por medio de ese cuerpo mi ego padece la acción del mundo y actúa en él. ¿Pero cuál es la relevancia de todo esto en el prospecto de la pregunta que nos inquieta, la constitución de la intersubjetividad? Pues bien, precisamente ese otro no es solo la experiencia de un psiquismo sino también una experiencia física, lo que permite emplazar el problema adecuadamente, arrancando, por una parte, desde la condición trascendental primera del ego y, por otra, del correlato que funge la presencia del otro a partir de su cuerpo como expresión física en el mundo. En el trasfondo mundano junto a todas las cosas del mundo, confundido entre ellas, se encuentra el cuerpo del otro. Y primeramente como cuerpo físico (Körper) entre las cosas que pueblan el mundo lo experimento antes del predicado otro.

El otro no nos viene dado inmediatamente en original, como sí mismo con sus vivencias, constituciones de mundo, síntesis judicativas, etcétera. Si así fuera, sería accesible inmediatamente en su totalidad constituyente como a mí mismo. Sería un "yo mismo". Ello resulta ciertamente impensable. Es allí donde la medianía del cuerpo del otro juega su rol. La presencia del cuerpo del otro en el mundo se encuentra con la trascendencia fundamental del ego en el mundo a partir de su cuerpo. Así el cuerpo del 
otro, primeramente como cuerpo físico (Körper), se ofrece como una experiencia dotada de una esencia eventualmente explicitable que no pertenece a mi esfera de yoicidad y que, no obstante, de ser explicitable, solo en mi subjetividad constituyente puede cobrar sentido y ser verificable. Solo a partir de mí y mi cuerpo orgánico propio es posible dar cuenta de ese cuerpo precisamente como un cuerpo orgánico otro (Lieb). Cuerpo y cuerpo. El uno y el otro en "carne y hueso" como señala sucesivamente Ricoeur (Ricoeur, 242). A mi cuerpo dado originalmente comparece un segundo cuerpo como percepción al que se refleja. Un analogon. Original enfrentado a un semejante. De mi cuerpo al otro, en la unidad de lo diverso, le asiste un irreflexivo deslizamiento de sentido: es cuerpo vivo y no objeto. De la representación de un cuerpo físico (Körper) en el mundo apresentamos un cuerpo vivo en el mundo (Lieb). Cuerpo que suministra el fundamento motivante para una exégesis espontánea e inevitable del cual obtiene su sentido. La apresentación no se trata de una inferencia, no es un acto de pensamiento ni una síntesis superior de la conciencia. Es una síntesis pasiva de la conciencia en la cual la transferencia de sentido desde el cuerpo físico como cosidad (Körper) al cuerpo vivo otro mencionado (Lieb) encuentra por base una similitud, una mímesis que opera como fundamento para una motivación analogizante denominada por Husserl como apareamiento (Paarung), a partir de la cual opera la transferencia de sentido referida. A la aprehensión anterior de un objeto, al que le sucede un segundo similar, le opera esta transferencia de sentido analogizante espontánea (Husserl, Meditaciones, 95). Esta forma originaria de síntesis pasiva de identificación también es denominada por Husserl en algunos pasajes como asociación. Así los cuerpos llegan a ser conscientes en un vivo evocarse el uno al otro, donde mi cuerpo orgánico presente en mi esfera de pertenencia de forma original opera como fundamento para realizar el deslizamiento de sentido al cuerpo orgánico otro. Es decir, un cuerpo otro "como el mío". De esta manera queda radicada una trascendencia intencional donde dos objetos distintos, mi cuerpo y el cuerpo otro, se evocan el uno al otro en coincidencias cuyo caso extremo es la igualdad.

¿Qué ocurre con la psiquis del otro? La apresentación de la psiquis del otro se da subordinada y entrelazada al cuerpo orgánico del otro. Es el comportamiento del cuerpo ajeno, el proceder bajo gobierno, las conductas corporales indicativas de sensaciones y de estados anímicos como, por ejemplo, el estar colérico, triste, alegre, nostálgico, ensimismado, pensativo, todos los cuales van mentando la existencia de un ego "otro" que es comprensible a partir de mis propias cogitaciones (Husserl, Meditaciones cartesianas, 157). Esta experiencia nos lleva a establecer que se trata de un cuerpo como el mío que se revela en señales indicativas de sucesos anímicos que se podrían traducir en un sistema de signos y expresiones; una verdadera gramática corporal (Husserl, Meditaciones, 151). Así el cuerpo orgánico indicaría lo psíquico apresentándolo por medio de una experiencia concordante y plenificante del comportamiento del cuerpo orgánico del otro. En caso de no ser así, no sería posible su apresentación o, en su defecto, en caso de no haber síntesis a partir de la unidad de concordancia e identidad en la experiencia del cuerpo orgánico del otro en su 


\section{Cristóbal Balbontín}

comportamiento o proceder, este podría ser advertido como pseudopsíquico. En esta accesibilidad verificable de lo que es originalmente inaccesible (el otro como horizonte infinito) se funda el carácter de lo extraño en cuanto existente, experiencia que no es plenificante inmediatamente y donde la "cosa" no es dada originariamente por lo que resulta concebible como análogo a mí y a partir de mí. En otras palabras, en mi monada se constituye apresentativamente otra monada en un curso de asociación constituyente que da cabida al modo otro en un cuerpo físico que cede lugar a la apresentación de un ego coexistente en el modo de su ser-ahí. La apresentación presupone, en consecuencia, un origen en la presentación.

La apresentación suministra, sin embargo, la anticipación vacía de una vida extraña. Es necesario que esta mención de sentido transferido se confirme por los signos concordantes que le confieren plenitud y estatus ontológico. Lo que aquí debemos tomar en consideración, entonces, es como se confirma la suposición de una vida extraña. Para ello Husserl se remite a la correspondencia de expresiones, gestos y comportamiento concomitante como confirmación de la anticipación de la vivencia del extraño. A dicha vía Husserl la denomina concordancia. A la anticipación del otro le es concordante su comportamiento. Un recurso a la evidencia que sigue a la "mención" sui generis del otro que se ha señalado. El otro ego, advertido como "gobernado psíquicamente" a partir de sus procesos sensibles, toma como base mi propio gobernar psíquico sobre mi cuerpo orgánico. Nuevamente, a partir de ello, cobra relevancia el cuerpo como sistema de signos. En este sentido el lenguaje, como señala Marie Andrée Ricard, no debe ser restringido al sentido lingüístico, sino a toda forma de comunicación comprometida con el cuerpo (89). La concordancia como resorte constitutivo permite una accesibilidad a lo primeramente inaccesible. Así, en consecuencia, a la apresentación le sigue y le concurre, como función constitutiva, el comportamiento concordante del cuerpo físico extraño que indica y confirma lo psíquico.

A los resortes mencionados le sigue un tercer factor concurrente a la constitución del otro. Por medio de la imaginación Husserl le da cabida, en último término, a la reflexión. Este nuevo elemento se mueve a través de variaciones libres de la conciencia por medio de las cuales se pueden concebir otros lugares y otras perspectivas. "Mi cuerpo físico orgánico, en cuanto referido a sí mismo, tiene su modo de darse del aquí central; todo otro cuerpo físico, lo mismo que el cuerpo físico del otro, tiene el modo del allí. En virtud de mis cinestesias, esta orientación el allí puede ser libremente cambiada. (...) Esto implica que yo, percibiendo a partir de allí, vería las mismas cosas, solo que en los modos de aparición distintos, que pertenecen al « ser yo mismo allí ». Es decir, que a toda cosa pertenecen constitutivamente no solo los sistemas de aparición de mi momentáneo a partir de aquí, sino también sistemas enteramente determinados correspondientes al de posición que me coloca en ese allí. Y así para cada alli' (Husserl, Meditaciones cartesianas, 154). Así con la ficción libero, en efecto, mi perspectiva y la transfiero a otra perspectiva, sin que por ello la vida del otro devenga jamás equivalente a la mía como única vida experimentada originariamente. Ella es tributaria del 
mecanismo de la apresentación que se nutre no solo de mi experiencia efectiva, sino también de la experiencia potencial.

Así se verifica una progresión que va del sentido vacío al sentido lleno, cuando del recurso analogizante, vía apresentación, pasando por la concordancia, llegamos hasta el concurso de la imaginación. Con ello se subraya nuevamente la posición de mi "ego" como norma, fundamento y medida para la constitución del otro. Por otra parte se ha intentado respetar la originalidad de la experiencia del otro que permita una trasgresión de la esfera monádica del ego cogito y la consecuente refutación del solipsismo denunciado. Para ello, nuestro cuerpo vivido desempeña, entonces, un papel decisivo en la forma que aprehendo al otro, no como cosa sino como subjetividad semejante $^{2}$. A diferencia de la sociología que parte de una colectividad como un hecho, Husserl realiza una progresión metódica que va desde el solus ipse a la comunidad con el objeto de identificar los hitos fundamentales que constituyen lo común.

Sobre el mundo, como horizonte predelineado de sentido, el alter ego me comprende a mí y obra sobre mí. Ello implica que este se trata de un mundo compartido con otros, comunizado (Vergemeinschaftung). La constitución del mundo en la conciencia resulta, entonces, en examen ante el otro. Con ello la presencia de los otros sella la condición de objetividad del mundo. Ahora bien, dicha impronta de objetividad impuesta por el otro opera primeramente como crisis de sentido. El otro, como otro ego, apresenta todo aquello que pertenece a la concreción de ese otro yo, su mundo primordial y su ego. Con ello el otro viene a ser una modificación de mí mismo. El otro no me deja indiferente, me pone a mí, sujeto tematizante del otro, en "entredicho". Las monadas están separadas y sus vivencias no conducen originalmente a las mías, ni las mías a las suyas. Así lo extraño en sí primero es el otro-yo a partir del cual se hace posible la constitución de un ámbito infinito de extrañeza y que desfonda al mundo al transferirle a aquel su misma extrañeza (Fremdheit). El mundo pasa a ofrecer una resistencia, un "pliegue" primitivamente inadvertido para su posible explicitación. Ya no se trata tan solo de un mero correlato de la conciencia, sino que el mundo es un correlato que pasa a ofrecer una resistencia a su aprehensión y convoca una aprehensión que se juega ante otros y que puede ser controvertida por ellos. "Por cuanto la subjetividad extraña surge por apresentación (dentro de la esencialidad propia exclusiva de mi subjetividad) con el sentido y la validez de otra subjetividad, con su esencia propia, se estaría inclinando a ver aquí, en un primer momento, un oscuro problema, a saber, ¿cómo puede realizarse una comunización, aunque sea en esta en esta primera forma de un mundo común? El cuerpo orgánico extraño, en cuanto aparece en mi esfera primordial, es mi unidad sintética $\mathrm{y}$, en consecuencia, en cuanto parte y

\footnotetext{
${ }^{2}$ Como lo resume felizmente Francisco Varela: "Es a través del cuerpo del otro que establezco un vínculo con ese otro primero como organismo semejante al mío, pero también percibido como presencia encarnada, lugar y medio de un campo experiencial. Esta doble dimensión del cuerpo (organismo/vivido; Körper/Leib) es un aspecto esencial de la empatía, camino real para acceder a la vida social consciente más allá de la interacción como intersubjetividad fundamental" (Varela, 251-252).
} 


\section{Cristóbal Balbontín}

determinación de mi esencia propia, inseparable de mí mismo. (...) Pero ¿cómo sucede que yo pueda hablar en general del mismo cuerpo, que aparece en mi esfera primordial en el modo del «allí » y en la suya y para él en el modo del «aquí»? Ambas esferas primordiales, la mía, que para mí como ego es la original, y la suya, que para mí es apresentada, ¿no están separadas por un abismo que yo realmente no puedo franquear, pues hacerlo significaría tener una experiencia original y no apresentativa del otro?" (Husserl, Meditaciones cartesianas, 159-160).

Así, el momento de la unidad entre presentación y apresentación del otro también es el recurso para una unidad posible de perspectivas con fundamento en un mundo en común. Ante la pérdida de la dictadura posible de un yo, surge la necesidad de conquistar un nuevo hablar como un nosotros que con su arbitraje ofrezca la posibilidad de una reconciliación con el mundo. Entre mi naturaleza dada primordialmente, como mundo particular, y aquella del otro apresentada se instaura entonces una coexistencia y una coincidencia posible que habilita un consenso implícito entre dichos mundos particulares. Se abre la posibilidad de un mundo común "consensuado" entre sujetos o intersubjetivo a partir de nuestra corporeidad mundana compartida. Justamente, a partir de esta naturaleza primordial y naturaleza presentificada, la experiencia del otro y su mundo relativo como lo extraño, cede lugar a una familiaridad en común. A partir de dicho mundo o naturaleza en común, Husserl radica también la solución del problema de la objetividad en tanto realidad compartida entre sujetos, es decir, intersubjetiva.

\section{CRÍTICA DE LA TEORÍA TRASCENDENTAL DE LA CONSTITUCIÓN DE LA INTERSUBJETIVIDAD ELABORADA POR HUSSERL}

Ahora bien, una de las inquietudes que saltan inmediatamente a la vista con la teoría de la constitución del otro en Husserl, es el recurso a una conciencia especulativa que a partir de sí y en sí aspira a constituir al otro. Un constituir que no es pasivo, que no es una acogida del ser del otro como dado, sino que es "productivo" o "generativo" del ser del otro en la conciencia del ego cogito. Ello suscita nuestra primera inquietud: la de saber en qué sentido se emplea la noción "constituir" a propósito del desarrollo formal del problema del otro en la Quinta meditación cartesiana en relación con los demás textos de Husserl, e identificar de este modo si el uso empleado del verbo sustenta la acusación de solipsismo e idealismo especulativo que habría en la resolución husserliana del problema del otro. El asunto cobra particular interés considerando que el verbo sustantivado "constitución" y el verbo "constituir" es de uso transversal y fundamental para toda la filosofia husserliana. Asimismo el asunto cobra importancia adicional, ya que Husserl, al establecer la esfera de la subjetividad trascendental como fundamento apodíctico para todo conocimiento posible, compromete, en la acepción que le asigne al rol constituyente de la subjetividad trascendental, una determinación ontológica, un nivel de compromiso con el idealismo e incluso la viabilidad de la totalidad de la empresa husserliana. 
Sorprendentemente, para un genio sistemático y científico como el de Husserl, la locución "constitución" y "constituir", a pesar de ser uno de los conceptos operatorios de su fenomenología, no tiene un uso terminológico preciso y distinto del lenguaje ordinario. Husserl mantiene, en consecuencia, un uso ambiguo de los términos a lo largo de toda su obra. Como bien lo señala Eugen Fink:

Pero ¿qué es constitución? ¿Dónde se define? Constitución, literalmente, quiere decir "poner junto" y puede entenderse evidentemente de diversas maneras: Puede significar "poner en orden", "producir" y "dar sentido de objeto a las cosas". En Husserl todas estas significaciones se mezclan cuando emplea el concepto de constitución en un sentido ingenuo y luego lo remite a un sentido nuevo, trascendental. Lo mismo vale para el concepto de producción. La vida de la subjetividad es caracterizada como productora. El ente viene siendo el resultado de producciones subjetivas. Las cosas son índices para la producción intencional. Tienen una relación necesaria con la subjetividad. No existen por sí mismas. ¿Pero cómo llevan las cosas a su existencia ¿Puede significar también edificar o realizar? (Fink, 203).

En el mismo sentido se pronuncia Roman Ingarden al señalar que: "Husserl a pesar de utilizar profusamente la palabra constitución no la toma de la tradición. El concepto general de constitución permanece indeterminado" (215). No obstante debemos admitir que se trata de un concepto que adquiere una relevancia específica en la obra husserliana y cuya determinación compromete una interpretación exitosa de su obra.

En Investigaciones Lógicas (Husserl, 2001) la función constituyente de la conciencia está claramente referida a la función dadora de sentido de la conciencia. Los entes son acogidos en la conciencia que opera, a su vez, como un referente de sentido para los entes. Una conciencia dadora de sentido. Así Husserl se refiere a la constitución del objeto como "dado en ciertos actos y nos es dado en ellos de la misma manera en la significación que lo mienta" (Husserl, Investigaciones Lógicas. Tomo I, 252). Los mismos términos son mantenidos en el Volumen II al señalar:

No será señalado nunca con bastante rigor el equívoco que permite llamar fenómeno no solo a la vivencia en que consiste el aparecer del objeto (...) sino también al objeto aparente en cuanto tal. (...) Es ya referencia del objeto fenoménico al sujeto fenoménico, al yo como persona empírica, que es naturalmente distinta de la inferencia del contenido de conciencia, en nuestro sentido de vivencia, a la conciencia en el sentido de unidad de los contenidos de conciencia (...) Una nueva referencia es la referencia objetivadora que establecemos entre la complexión de sensaciones vividas en el fenómeno y el objeto aparente; como cuando decimos que en el acto de aparecer es vivida la complexión de las sensaciones, pero es a la vez "aprehendida" "apercibida" de cierto modo y que en este carácter fenomenológico de la aprehensión animadora de las sensaciones, consiste en lo que llamamos el aparecer del objeto" (Husserl, Investigaciones lógicas Tomo II, 478). 


\section{Cristóbal Balbontín}

Más adelante expresa:

(...) en el juicio yo soy la evidencia que pende de cierto núcleo que hay en la representación empírica del yo y que no está definido con rigor conceptual. Si preguntamos ahora que sea lo que puede entrar en este núcleo conceptualmente impreciso y por ende inefable, que sea lo que constituya en cada caso, con seguridad evidente lo dado en el yo empírico (...) No solo es evidente el yo soy sino incontables juicios en la forma yo percibo esto aquello, siempre que en ellos yo no suponga meramente sino que esté seguro, con evidencia, de que lo percibido está dado tal como es mentado, siempre que yo aprehenda la cosa misma como es (482).

Y asimismo lo refrenda en algunos pasajes más adelante: "El núcleo fenomenológico del yo empírico está formado por actos que traen a la conciencia el objeto (...)" (486).

Ese tenor se mantiene incluso en los primeros pasajes de Ideas $I$ donde se señala que "el objeto intencional no es solo en general objeto de la conciencia, presente a la mirada del espíritu que está dirigido a él, sino que el objeto es aprehendido, advertido". (Husserl, Ideas relativas, 84). En el mismo sentido en un pasaje posterior: "Es pues un error creer que la percepción no se acerca a la cosa misma" (97). Y asimismo: "La percepción (...) aprehende un ello mismo en su presencia, en persona" (99). Así, al comienzo de la obra husserliana constitución significaba la clarificación de la estructura de sentido de la vida consciente por un "acoger", un "aprehender" o "advertir" lo que se manifiesta en ella en tanto fenómeno.

Sin embargo, como lo afirma el Shinji Hamazu, "de manera imprevista me parece a mí, la idea de constitución, relacionada a una explicitación del sentido del ser, pasa a una fundación de la estructura del ser en la subjetividad trascendental; ha cambiado desde una explicación a una creación" (Hamazu, 5). Pero ¿qué tan imprevisto puede resultar ese giro husserliano? Existen dos indicios que explican el cambio en la noción de constitución que también pueden ser diagnosticados como momentos o giros en la fenomenología husserliana.

Primero la introducción de la epoye como resorte de análisis metodológico concentra la mirada en el análisis de la legalidad constituyente de la conciencia y, a su vez, de la realidad y sus distintos fenómenos en la conciencia. Al efectuar la puesta en paréntesis del ser del mundo a través de la epoye, lo que hace Husserl es enfocarse en una conciencia inmanente, en un ego puro que resalta la participación de la conciencia en la génesis de la realidad en la conciencia y sus fenómenos. Esta opinión es compartida por Leslie Beck que en el Coloquio de Royaumont resaltó la relación entre epoye y la mutación de sentido del concepto de constitución (Beck, 317). Así por ejemplo en Ideas $I$ ya es posible comprobar un giro en la noción de constitución después de la epoye: "La reducción del mundo natural al absoluto de la conciencia da por resultado ordenes fácticos de vivencias de conciencia de ciertas formas sujetas a reglas señaladas en las que se constituye, como correlato intencional, un mundo 
morfológicamente ordenado" (Husserl, Ideas relativas, 133). Y luego señala: "De nuestras consideraciones podemos sacar la tesis eidéticamente valida y evidente (a propósito de la corriente unitaria de las vivencias como idea) de que ninguna vivencia concreta puede pasar por independiente en sentido plenario. Todas ellas están 'menesterosas de complemento' por parte de un complejo no arbitrario sino forzosamente determinado en su índole y forma (...)" (197). Luego se refiere específicamente al problema de la constitución en el parágrafo número ochenta y seis titulado Los Problemas funcionales: "Pero los problemas mayores de todos son los problemas funcionales o los de la "constitución de objetividades en la conciencia". Conciernen a la forma en que, por ejemplo, con respecto a la naturaleza, la noesis, animando lo material y entretejiéndose en continuos y síntesis múltiplemente unitarios. "Es de un cabo a otro 'conciencia' fruto de toda razón y sin razón, de toda ley e ilegitimidad, de toda verdadera realidad y ficción, de todo valor y contravalor" (207).

Ahora bien, la segunda razón la expone Walter Biemel en el mismo coloquio de Royaumont al señalar que, a propósito del desarrollo del texto de las Dingvorlesung de 1907, Husserl se encuentra con la necesidad de desarrollar el concepto operatorio de la epoye, que aquí por primera vez se extiende a la constitución de la cosa (Biemel, 1957). Ello coincide con el desarrollo llevado a cabo de la noción del tiempo en el escrito Vorlesungen zur Phänomenologie des inneren Zeitbewusstseins de 1904/1905. En dicho desarrollo Husserl se concentra en el análisis del tiempo inmanente de la conciencia. En el análisis en cuestión descubrirá que el tiempo no solo es el sustrato de pasividad de la conciencia que hace posible a la misma conciencia y todo fenómeno en la conciencia, sino que además el funcionamiento de la intencionalidad misma está perfectamente articulada con el ordenamiento del tiempo de tal manera que presente, pasado y futuro son expresiones de la intencionalidad y su movimiento que pueden operar como inflexión activa sobre un presente constituyendo un ahora que deslinda en el futuro y el pasado inmediato que Husserl denomina retención y protensión, respectivamente, y que permiten la posibilidad y persistencia de un presente, que no es otra cosa que la actualidad de dicha intencionalidad. En Husserl el descubrimiento de que toda la estructura del tiempo inmanente tiene una correspondencia exacta con la intencionalidad va a provocar una honda impresión y va a repercutir en la lectura de la conciencia constituyente de los objetos a la luz de las leyes estructurales del tiempo, con lo que el rol de la subjetividad se ve marcadamente reforzado. Como señala Van Peursen: "El tiempo permite la constitución de fenómenos como tales, pero implica entonces una creación fundamental que escapa a los conceptos. Este núcleo poco accesible del pensamiento husserliano representa su idealismo. No se trata simplemente de una creación del mundo y sus objetos por un sujeto absoluto. La operación intencional se efectúa en el horizonte del tiempo" (Van Peursen, 197). Por su parte Ricoeur ha advertido aquí un punto de inflexión de la fenomenología husserliana al señalar que: "La interpretación de la presencia como presente hace depositar sobre la teoría de la temporalidad la totalidad del peso de los problemas de la constitución" 


\section{Cristóbal Balbontín}

(194). Además, y a propósito del desarrollo de la noción del tiempo, Husserl se adentrará, en el contexto de la realidad natural y anímica en Ideas II, en las nociones de protensión, imaginación y apresentación. Todas ellas son formas de participación activa de la conciencia en la constitución de las cosas. La conciencia se anticipa para ir más allá de lo dado para formar una unidad en la conciencia. El clásico ejemplo de que nunca tenemos una figura espacial dada en todos sus escorzos a la vez implica y compromete a la conciencia en una función activa que, a partir de los escorzos dados, apresenta sus otros lados. Ello revela un ahondamiento en la concepción de la función creativa de la conciencia en la constitución del objeto. Lo anterior es confirmado por Eugen Fink que señala al efecto: "Igualmente el rol constitutivo de la imaginación y la constitución del tiempo en Husserl apunta en este sentido. La interpretación que acuerda a la intencionalidad una producción de ser y toma las intuiciones como residuos para la función constitutiva corresponde a la perspectiva 'trascendental idealista' a la que se acerca en sus últimos años" (Fink, 320).

Así, como señala Biemel, la conciencia comienza a ser considerada el constituyente tras toda constitución (47). Husserl decide adentrarse entonces en la ipso constitución. Ello se refleja bien en la carta del 5 y 6 de julio de 1911 enviada a Dilthey en la época en que Husserl ya pensaba en el desarrollo del método fenomenológico propiamente tal con miras a la publicación de Ideas I dos años más tarde: "Creo que por detrás de los problemas del ser entendidos de manera completa, es decir, incluyendo igualmente la 'constitución' del ser en la conciencia, no puede haber otro problema que tenga significación (...) Hablar de un ser que estaría detrás y sería incognoscible es por principio absurdo"(Husserl citado por Biemel, 47). Ello se corresponde con una nota en el diario de Husserl, con fecha 25 de octubre de 1906, donde revela la necesidad de una crítica de la razón (Husserl citado por De Waelhens, 131). Marca con ello un mayor interés por Kant que irá creciendo con el tiempo y coincide con el uso de la noción constitutiva de la conciencia en un sentido más activo. En efecto, en esta época Hussserl habría mantenido una correspondencia con Natorp, de la escuela de Marburgo, que marcaría este giro en su filosofía (Biemel, 28). De alguna manera la advertencia kantiana acerca de una disyuntiva entre idealismo y realismo, que debe ser resuelta en uno u otro sentido, comienza a preocupar al espíritu de Husserl ${ }^{3}$.

Así a partir de esta época y de manera creciente, como indica el profesor Hamazu, se va a observar un cambio en el uso del verbo aufbauen (constituir) desde la forma pasiva señalada, pasando por una forma reflexiva, sich konstituieren, hasta una forma activa con la consecuente introducción del verbo sichbilden (autoconstituir o constituir a sí mismo) (Hamazu, 5). El uso reflexivo del verbo va a encontrar expresión en Ideas II y III y el uso activo a su vez en el desarrollo posterior a dichos textos. Así

\footnotetext{
${ }^{3}$ Hay que recordar que el mismo Kant, en la sección "Deducción trascendental de los conceptos del entendimiento" a propósito de la condición de posibilidad de aplicación de las categorías a los objetos de la experiencia, se plantea el problema sobre las condiciones que deben ser cumplidas por los contenidos de apariencias para ser dados como objetos (Kant, 1992).
} 
mientras en el primer Husserl de las Investigaciones Lógicas, anterior a la epoye, la conciencia dadora de sentido y significado representa una clara expresión de un Husserl más realista, el Husserl de fines de los años 1920-1930 se acerca a un progresivo idealismo. Ello es confirmado por Roman Ingarden, que no obstante a la sazón advierte: "La cuestión se vuelve a plantear, a la de saber que acción efectiva hay que acordar a la intencionalidad de la conciencia pura y en qué sentido y en qué medida esa intencionalidad es, según Husserl, 'productora de ser'. Husserl nunca se expresó de manera definitiva y segura sobre este punto y a lo largo de su vida dudo de la decisión a tomar. Si bien tendió cada vez más a reconocer a la intencionalidad de la conciencia un rol productor de ser" (Ingarden, 228). Paradójicamente en la noción de mundo de vida, Lebenswelt, a propósito del escrito tardío de la Crisis de las ciencias europeas y la fenomenología trascendental es posible encontrar la noción de mundo como correlato y forma de relación fundamental de la conciencia con el ser del mundo, operando una recuperación de la noción de constitución en el sentido pasivo que había inaugurado el primer Husserl: "Cualquier cosa que experimentamos, con la que tratamos habitualmente (...) se da a sí misma, advirtámoslo o no, como cosa en el mundo en el respectivo campo perceptual" (Husserl, La crisis, 262). Así, del modo en que opera el método fenomenológico, por medio de un movimiento pendular que va desde la intuición y sus modos (noema) hasta remontar a la conciencia intencional y su estructura dadora de sentido (noesis) y volver a descender sucesivamente, así la obra del mismo Husserl sigue el mismo sentido pudiendo advertirse un primer Husserl más intuicionista que asciende a la subjetividad con un creciente sentido idealista, como expresión de un segundo Husserl, para volver a descender al final de su vida y recuperar el sentido intuicionista como habilitación del mundo trascendente del Lebenswelt como sustrato fundamental de la conciencia. A raíz de ello Walter Biemel señala que "durante su vida Husserl experimentó una gran dificultad para constituir en sistema la enorme cantidad de enfoques y materiales que acumulaba" (54).

Pero no perdamos nuestro hilo central de desarrollo. Como señalamos, en el movimiento ascendente descrito en la obra husserliana hacia 1931 se engendra la publicación de las Meditaciones cartesianas a propósito de las conferencias dictadas por Husserl en París. En ella es manifiesto el momento idealista por el que atraviesa la obra husserliana con la consecuente comprensión del rol constituyente de la conciencia como "creadora". Las Meditaciones cartesianas, como su nombre lo indica, se proponen examinar la posibilidad de establecer un fundamento apodíctico para las ciencias a partir del cogito cartesiano. En dicho ejercicio Husserl llega tempranamente a la conclusión de que el ego cogito debe ser radicalizado frente a la comodidad del argumento de la res extensa introducido por Descartes para acreditar el ser del mundo. La grandeza de Descartes, según Husserl, es el haber realizado el proyecto de la filosofía como ciencia que a la vez operará como fundamento para las ciencias. Pero Descartes traiciona su radicalismo al tomar el ego cogito como primer anillo de una cadena deductiva que va de la res cogitans a la res extensa avalada por el argumento que acredita la existencia de 


\section{Cristóbal Balbontín}

Dios. En dicho gesto queda comprometido un dilema entre una filosofia donde la subjetividad es polo de referencia de todo lo expresable y donde, a la vez, Dios es polo de referencia de todo lo existente. La razón introductoria de las Meditaciones cartesianas es entonces destruir este sentido original del cartesianismo que lo lleva a la noción de infinito. La fenomenología trascendental, en consecuencia, asume con coraje las enormes dificultades que iremos a ensayar de una filosofia que se concentra en ser una egología y no en una ontología, de una filosofía donde el ser no es aquello que da peso de realidad al objeto más sobre todo que dicha realidad se funda en el ego cogito en tanto realidad que es mentada por la conciencia. Así el ejercicio de las Meditaciones cartesianas parte por hacer del ego cogito el principio real, el punto de partida real de la filosofia con el propósito fundacional absoluto para las ciencias. La primera evidencia del ego cogito subordinará a él la presencia del mundo. La introducción de la epoye viene a fortalecer y a justificar, en consecuencia, la existencia del mundo en y para mi conciencia, lo que repercute en el hundimiento de un posible suelo ontológico como fundamento para el conocimiento. La epoye de las Meditaciones cartesianas, en este sentido, no es un mero poner entre paréntesis sino la expresión del mundo con todas sus modalidades como fenómeno y, en tanto fenómeno, en y para la conciencia. Dicho de otro modo, la epoye no pretende aflojar un lazo ontológico para asegurarlo mejor, sino en disipar sin retorno la ilusión realista del en sí. De esta manera, la empresa de las Meditaciones cartesianas es una empresa gnoseológica. Y dicha preocupación gnoseológica no está entrelazada a otra preocupación que la sobrepase. "Yo no puedo actuar y portar el juzgamiento de valor en un mundo otro que aquel que encuentra en mí mismo y obtiene de mi su sentido y validez" (Ricoeur, 168). Así la cuestión ontológica se desliza indirectamente a propósito de la cuestión gnoseológica. En lo particular, en la Segunda meditación cartesiana hay un desplazamiento del centro de gravedad de la evidencia trascendental del ego cogito-cogitatum a una exégesis destinada a la elucidación de las cogitaciones múltiples de la que es capaz este ego. El ego es el polo de todas las cogitaciones y todas ellas resultan integradas a este ego. Así la fenomenología será un análisis de doble título distinguiéndose a título de una cogitatio y a título de un cogitatum. Así la vida del ego no es un estallido anárquico sino una guía que responde a la unidad del ego como fuente de existencia, orden y sentido del mundo.

En la Tercera meditación cartesiana se examina la situación de la evidencia en el idealismo fenomenológico. La evidencia, según Husserl, es la presencia de la cosa misma en original (por oposición a una presentificación, souvenir, retrato, imagen, símbolo, signo o concepto, por ejemplo). Es la presencia, como señala Ricoeur "en carne y hueso" (Ricœur, 242). La evidencia o die Selbstgegebenheit que Husserl considera originaria. Ahora bien, si el objeto está constituido por perfiles y perspectivas, algunas invisibles, ¿Qué es lo originario? ¿Cómo la unidad del objeto puede ser otra cosa que una unidad producida? La noción de presencia en chair et os parece introducir un factor disparatado, un en sí del objeto que llena un vacío. Una interpretación idealista, en este caso, del "sentido" mina la posibilidad de un sí mismo de la cosa. Es 
necesario tener en consideración que la fenomenología de Husserl es trabajada por dos exigencias que esta meditación tiende a armonizar. De un lado, una exigencia idealista que se expresa en el tema de la constitución y que no es más que un proceso de verificación cotidiana del curso de la conciencia, un hacer de la conciencia, y, por otro lado, una exigencia intuicionista primera a la reducción fenomenológica que se expresa en el aforismo "zu den Sachen Selbst!" y que determina la obra del conocimiento hacia un acoger la cosa misma que se da. Así la unidad de sentido es presumida por la obra de la conciencia anticipante y, por otra parte, confirmada por la sanción de lo manifiesto. Esta interpretación moderada de la fenomenología husserliana es sacrificada por el idealismo acentuado de las Meditaciones cartesianas que termina subordinando la evidencia a un idealismo trascendental. "Toda justificación procede de la evidencia que encuentra su fuente en esta subjetividad trascendental. Es en esta síntesis donde ella encuentra su fundamento trascendental último" (Husserl, Meditaciones cartesianas, 183). Este giro en la teoría de la evidencia es decisivo. Toda tentación de identificar la intencionalidad con un contacto con el ser es descartada, la evidencia se reintegra en mí como un momento de mi monada. Así resulta ajustada la evidencia a la exigencia de una egología. Esta tiende a desmitificar el aspecto absoluto del ser. En consecuencia, la noción de una síntesis de una presencia total no es más que una idea. A este precio la teoría de la evidencia entra en una egología. La importancia futura de la Quinta meditación cartesiana se anuncia, por lo tanto, de una manera considerable porque si la evidencia no nos hace salir de nosotros mismos no habrá propiamente alteridad en todo el sentido del término. La Cuarta meditación cartesiana no hace una exégesis del ego a partir de la cogitatio, sino que vuelve la mirada sobre el ego. El ego es el polo idéntico en la multiplicidad de actos, en las diversas cogitaciones, es correlato de todas las cogitaciones múltiples. Así, en este apartado el ego no se muestra solo como un polo de referencia más como sustrato de propiedades permanentes. El yo tiene en sus variaciones propiedades permanentes a mismo título que ante la variación de una cosa como ante la variación de circunstancias, de tal forma que opera de forma fija determinado por el hábito. Una verdadera colección de tomas de posición.

De este modo las tres primeras meditaciones desarrollan la idea de que el sentido del ente se obtiene en y para la subjetividad trascendental, mientras que la Cuarta meditación cartesiana se ocupa de la constitución del ego mismo. En ese gesto filosófico, en tanto que interpretación idealista de su fenomenología, Husserl aborda el tema de la constitución del otro con la pretensión de reducir toda alteridad a la vida monádica del ego. La decisión de abordar el problema de la alteridad en un momento de pronunciado idealismo conlleva que el intento de Husserl fracase.

En efecto, la teoría trascendental de la experiencia del extraño arranca con la irrupción de la objeción del solipsismo. Esta objeción es la que denuncia el sentido común: no procede reducir a los otros a la apresentación que tengamos de ellos. Tal objeción, dice Ricoeur, debe ser reconocida como una aporía de partida por la fenomenología. ¿Por qué dudar del ser de los otros que nos son dados fácticamente 


\section{Cristóbal Balbontín}

como alter ego desde nuestro primer momento en este mundo? Esto es consecuencia de alguna manera del solipsismo fenomenológico desarrollado en las Meditaciones cartesianas que alcanza su punto más álgido en la Cuarta meditación cartesiana. Ello implica obligar a la fenomenología trascendental, que no puede realizar ninguna descripción, ninguna explicitación, ninguna constitución sin un objeto que sirva de guía de la experiencia trascendental. En este caso la paradoja es pretender que no sea la experiencia del otro la guía trascendental, sino el cuerpo del otro, y aún antes que ello, cuerpo que comparece primeramente como cosa en el mundo a la conciencia. Ello obliga a recurrir al resorte de una segunda epoye de lo ajeno que ya hemos descrito en capítulos anteriores de este trabajo. Sin embargo, dicha misma epoye ostenta una serie de dificultades.

En efecto, en dicha epoye puede advertirse una petición de principios, pues se hace abstracción de una determinación de lo ajeno que no obstante ya está impreso en el cogito como determinación o característica precisamente de lo ajeno cuya constitución se busca establecer. En palabras sencillas, la respuesta está planteada en la misma pregunta. Asimismo, siguiendo a Alfred Schütz y su intervención en Royaumont, la epoye encuentra un contrasentido al pretender hacer una epoye de lo ajeno y, por otro lado, se le conserva en la esfera de pertenencia como base de su análisis (Schütz, 302). Ello se hace extensivo al mecanismo de la concordancia en la constitución del otro. ¡Cómo es posible el recurso a la concordancia si en la epoye se ha hecho abstracción de toda experiencia de subjetividad ajena a partir de la cual se pretende atribuir una concordancia posible entre manifestaciones corporales y psiquis! Esto confirma el fracaso de la segunda epoye metodológica, ya que no se lleva a cabo ni puede ser llevada a cabo con la radicalidad anunciada. Sorprende que Husserl insista en esta segunda epoye no obstante las evidentes falencias metodológicas que conlleva. En dicho sentido, el recurso a la epoye constituye además la llamada falacia de la excavación en tanto viene a deformar por medio de una ficción precisamente la experiencia de la alteridad que se intenta dilucidar. Agreguemos a ello que la concordancia presupone tipos de normalidad de comportamiento del otro que solo pueden encontrar su fundamento en las acciones y correlaciones funcionales de la subjetividad ajena que debieron haber sido eliminadas por la epoye. De este modo la noción de la epoye o el recurso a la concordancia necesariamente falla.

Luego, el programa de la Quinta meditación cartesiana avanza para poner su mirada sobre el cuerpo propio que servirá de base para una cadena de significaciones que llevará del ego propio al alter ego. A partir de la segunda reducción se ha buscado obtener este cuerpo propio como nexo primordial con la naturaleza a partir de la cual operar una transferencia de sentido que vaya de mi cuerpo a la otredad presente en mi esfera de percepción como cuerpo ajeno basándose en la unidad sobre lo diverso, que es el recurso de la analogía como expresión de una síntesis de la pasividad de la conciencia. Sin embargo, ello es objetable. Primero la experiencia del cuerpo propio, de la carne como propia no es una realidad, ni una constatación inmediata. Reconocemos, 
en efecto, que es fuente de una abstracción al confrontarlo con un cuerpo ajeno. El cuerpo es primeramente el cuerpo, soy cuerpo antes de atribuírmelo como "mío" por oposición al cuerpo ajeno. Por lo tanto, donde se busca una experiencia inmediata de lo propio hay más bien una exégesis que permanece tributaria de una abstracción en la que ha tomado rol activo lo ajeno en su definición aún antes de su "debida" constitución. A mayor abundamiento, Husserl presupone una semejanza entre el cuerpo ajeno, percibido exteriormente, y el cuerpo propio, percibido interiormente por medio de cinestesias (sensaciones de movimiento muscular), cenestesias (sensaciones viscerales) y ubistesias (sensaciones localizadas). ¿No hay acaso una ficción en la pretendida analogía de los cuerpos? Además frente a un cuerpo de una condición radicalmente distinta, por ejemplo un cuerpo mutilado o deforme, se sugieren dificultades para que opere la analogía en la pasividad de la conciencia según la forma descrita. Si bien es cierto que Husserl se refiere a anomalías como la ceguera, la sordera, etcétera, no se pronuncia sobre casos tan extremos. Estas dificultades que puede presentar la analogía como fundamento para la apresentación de la psiquis del otro han sido resaltadas ya por Scheler, Sartre y Merleau Ponty.

Ahora bien las siguientes dificultades, y quizás las más emblemáticas de la teoría de la intersubjetividad husserliana, las encontramos en el siguiente paso que hace Husserl en el recurso a la apresentación y a la imaginación. Por su parte, con la imaginación habíamos dicho cohago la posición del otro "como si estuviera allí" imaginando el mundo como se percibe desde su posición con la consecuente ficción de su posible mundo particular constituido. Ella es tributaria del mecanismo de la apresentación que se nutre no solo de mi experiencia efectiva, sino también de mi experiencia potencial (Husserl, Meditaciones cartesianas, 119). Estos dos "resortes" constitutivos a los que remite Husserl están en el corazón de este creciente idealismo denunciado y a la pretendida función creativa de la conciencia. No obstante que Husserl expresamente desea resolver la crítica del solipsismo que se le ha atribuido, resulta realmente conflictiva la pretensión de que a partir del a priori universal, que abarca una infinita diversidad de tipos de actualidades y potencialidades imaginables según las leyes propias de la motivación en la forma universal de la temporalidad de la conciencia inmanente, se pueda albergar la respuesta "qué es el otro". ¿Puede siquiera efectivamente pensarse en otros egos trascendentales considerando que solo el ego cogito puede pensarse en singular y como originario para mí mediante la duda cartesiana? Desde un comienzo los otros estarían siempre cubiertos de un velo de dubitabilidad. El problema lleva a amarrar el conocimiento objetivo en su punto de partida al ego trascendental de la reducción fenomenológica, lo que lo lleva a recaer en un subjetivismo solipsista desde donde todo pasaje a una subjetividad ajena fuerza una metafísica de corte leibniziana ${ }^{4}$. Donde, además, resulta ambiguo que Husserl no incurra del todo en una operación deductiva del otro a partir del ego cogito que expresamente quiso evitar y que condenaría directamente la fenomenología a un

\footnotetext{
${ }^{4}$ Ficción que hace recordar el gesto metafísico de una armonía preestablecida de monadas en Leibniz.
} 


\section{Cristóbal Balbontín}

idealismo absoluto. De todas formas, con ello se fuerza una prioridad ontológica del sujeto sobre el otro que resulta aporética considerando que el alter ego ostenta como tal un estatuto ontológico a lo menos simultáneo. No resulta comprensible un alter ego de una menor jerarquía ontológica salvo un amigo imaginario. En consecuencia, no se ve cómo la transferencia de sentido, vía apresentación e imaginación, puede implicar la producción de la vida psíquica del otro. Lo cierto es que la condición de ser psíquico se produce a partir de la apresentación y se ve enriquecida por la imaginación. Metodológicamente antes de eso hablamos de un ente en tanto cuerpo. Pero ello debe ser denunciado. No es posible la experiencia original de los estados psíquicos ajenos a partir de los estados psíquicos propios. En ello hay una contradicción en los términos. Así, con dicho recurso Husserl en vez de salvar la acusación de solipsismo solo termina profundizándola. Estos problemas se prolongan y extienden hasta la constitución de la intersubjetividad y con ello al problema de la objetividad y el mundo espiritual. No resulta claro, asimismo, como la apresentación del otro a partir de su cuerpo puede conllevar la apresentación de su mundo primordial, otro mundo primordial que mediante síntesis de identidad permita identificar un mundo en común o "mundo entre mundos primordiales". ¿Cómo mediante la intrafección experimentar el mundo del otro? Resulta imposible. Una distancia irreparable se dibuja que solo cede lugar a una experiencia imaginativa.

Como habíamos visto, el siguiente paso que da Husserl en su teoría trascendental de la experiencia del extraño será recurrir a la concordancia. Como habíamos visto Husserl la comprende como el recurso a la correspondencia de expresiones, gestos y comportamientos concomitantes como confirmación de la anticipación vacía de la vivencia del extraño por medio de la apresentación. Sin embargo, el resorte de la concordancia también exhibe una serie de dificultades. La concordancia corresponde a un momento pasivo de la constitución que compromete el problema del lenguaje y la noción de la evidencia desarrollada por Husserl. Los comportamientos corporales del otro son indicativos, índices, significantes de sucesos psíquicos significados. Pero ¿cómo es posible entender su comportamiento desde la perspectiva del lenguaje si por vía de la epoye se ha hecho abstracción de toda noción de subjetividad ajena? Asimismo, ¿no es acaso cierto que el interpretar la conducta de los otros, lo hacemos a partir de un repertorio completo de significados que vienen dados por la cultura en que estamos insertos y precisamente de la que se ha hecho abstracción? Ello repercute en que la concordancia presupone tipos de normalidad del comportamiento del otro que solo puede encontrar su fundamento en las acciones y correlaciones funcionales de la subjetividad ajena y la cultura que fueron eliminadas por la epoye. Como bien lo señala Alfred Schütz, en un sentido más profundo todas las modalidades de la subjetividad ajena, sea hombre, niño, mujer, anciano, extranjero, remiten a una sedimentación de un estándar de comportamiento y a un significado cultural (Schütz, 302). Si no hay una "norma", ni subjetividad ajena ni cultura después de la epoye del tipo para interpretar una conducta, no es posible recurrir a la concordancia. La misma crítica se puede hacer 
en la perspectiva de la evidencia. El recurso a la concordancia es el recurso a la evidencia en tanto que se aspira a que el suceso psíquico anticipado encuentre correspondencia con la presencia original de un comportamiento corporal determinado. Pero ello es inviable desde la perspectiva de la epoye realizada y desde la perspectiva de la totalidad de las Meditaciones cartesianas. Como ya vimos la Tercera meditación cartesiana se encarga de deformar el concepto de evidencia heredado de las Investigaciones lógicas y termina subordinándolo a un idealismo trascendental donde toda justificación procedente de la evidencia encuentra su fuente en esta subjetividad trascendental última. En consecuencia, las mismas Meditaciones cartesianas empiezan a fracturarse. De ello se siguen dos consecuencias: por un lado la única manera de interpretar el comportamiento corporal en correlación con un suceso psíquico a partir de la epoye es tomando por base mis propias correlaciones entre cuerpo y psiquis, lo que significa reducir al otro a un reflejo de mi ego con una leve modificación dada por la asimetría de su posición. Pero ello ya está lejos de la constitución de otro que precisamente se encuentre en una radical alteridad en tanto otro. Con ello la egología se ve profundizada. Y, segundo, la epoye nuevamente resulta desbordada por el lugar eminente que le corresponde al lenguaje en la constitución del otro que no es formalmente rescatado por Husserl en estos pasajes. Ello revela a su vez que dentro de la misma Quinta meditación cartesiana, por vía de la concordancia, está introducida la noción de constitución en un sentido pasivo, como un "dar sentido" al comportamiento corporal del extraño. En consecuencia el rol constitutivo de la conciencia en sentido activo y en sentido pasivo en la misma Quinta meditación cartesiana encierra una contradicción que revela la tracción a la que está sujeta la Quinta meditación, como dice Ricoeur, entre dos experiencias: restituir el otro en mí y constituirlo como otro. Paradoja que está latente en todas las Meditaciones y en toda la fenomenología husserliana, como lo señalamos con anterioridad. La insistencia en el desarrollo mismo de un idealismo y un realismo llaman a que la Quinta meditación estalle sin que su respuesta sea satisfactoria, comprometiendo, quizás, a la totalidad de la fenomenología husserliana.

\author{
Universidad Andrçes Bello* \\ Facultad de Humanidades y Ciencias Sociales \\ Fernández Concha $N^{\circ} 700$, Santiago (Chile) \\ cbalbonting@gmail.com
}

\title{
OBRAS CITADAS
}

Beck, Leslie. El problema de la intersubjetividad trascendental en Edmund Husserl. Ponencia presentada en el Coloquio de Royaumont. Título original: Husserl, Cahiers de Royaumont (Trad. Amalia Podetti). Buenos Aires: Paidos, 1957: 317-323. 
Biemel, Walter. Las fases decisivas en el desarrollo de la filosofia de Husserl. Ponencia presentada en el Coloquio de Royaumont. Título original: HUSSERL, Cahiers de Royaumont (Trad. Amalia Podetti). Buenos Aires: Paidos, 1957: 47- 72.

De Waelhens, A. Sobre la idea de la fenomenología. Ponencia presentada en el Coloquio de Royaumont. Título original: Husserl, Cahiers de Royaumont (Trad. Amalia Podetti). Buenos Aires: Paidos, 1957: 131-156.

Fink, Eugen. Los conceptos operatorios de la Fenomenología. Ponencia presentada en el Coloquio de Royaumont. Título original: Husserl, Cahiers de Royaumont (Trad. Amalia Podetti). Buenos Aires: Paidos, 1957: 203-215.

Hamazu, Shinji. Identity and Alterity, Shutz and Husserl on the phenomenology of intersubjectivity. 2010. Disponible en: http://www.cuhk.edu.hk/rih/phs/PEACE/papers/ShinjiHAMAUZU.pdf

Husserl, Edmund. Investigaciones Lógicas Tomo II (Trad. Manuel García Morente y José Gaos). Madrid: Alianza, 2002.

_ Investigaciones Lógicas Tomo I (Trad. Manuel García Morente y José Gaos) Madrid: Alianza, 2001.

_ La Crisis de las Ciencias Europeas y la Fenomenología trascendental, (Trad. Jacobo Muñoz y Salvador Mas). Barcelona: Crítica, 1990.

— Meditaciones cartesianas. (Trad. Mario A. Presas). Madrid: Ediciones Paulinas, 1986.

_- Ideas relativas a una fenomenología pura y una filosofía fenomenológica Libro I (Trad. José Gaos). México: Fondo de Cultura Económica, 1962.

Ingarden, Roman. El problema de la constitución y el sentido de la reflexión constitutiva en Husserl. Ponencia presentada en el Coloquio de Royaumont. Título original: Husserl, Cahiers de Royaumont (Trad. Amalia Podetti). Buenos Aires: Paidos, 1957: 215- 240.

Kant, Immanuel, Crítica de la razón pura, (Trad. José del Perojo y José Rovira Armengol). Madrid: Losada, 2003.

Merleau-Ponty, Maurice, Phénoménologie de la perception, Paris : Gallimard, 1976.

Ricard, Marie-Andrée. L'empathie comme expérience charnelle ou expressive d'autrui chez Husserl. Recherches Qualitatives (2005), Vol 25.

Ricœur, Paul. A l'école de la phénoménologie. Paris: Librairie Philosophique J. Vrin, 1998.

Schütz, Alfred, El problema de la intersubjetividad trascendental en Husserl. Ponencia presentada en el Coloquio de Royaumont. Título original: Husserl, Cahiers de Royaumont (Trad. Amalia Podetti). Buenos Aires: Paidos, 1968: 291-316.

Van Peursen, C.A. La noción de tiempo y ego trascendental en Husserl. Ponencia presentada en el Coloquio de Royaumont. Título original: Husserl, Cahiers de Royaumont (Trad. Amalia Podetti). Buenos Aires: Paidos, 1957: 197-203.

Varela, Francisco, El fenómeno de la Vida. Santiago: Dolmen, 2000. 